\title{
Research on the Effectiveness of Modelling of Autonomous Flight System for Monitoring Agricultural and Industrial Sites
}

\author{
Nayden Nenkov ${ }^{1, *}$, Nedyalko Domitrov ${ }^{1}$ \\ ${ }^{1}$ Konstantin Preslavsky University of Shumen, College in Dobrich, Bulgaria
}

\begin{abstract}
Unmanned systems (drones) have been widely used for both military and civilian purposes for many years. Knowing the capabilities of the different systems allows for the right selection in preparation for a specific task, and each task requires the correct selection of the flight system, scientific and navigation equipment. The application of these systems has grown enormously in various fields such as: leisure, information and media, monitoring and inspection (electrical, pipelines, industrial installations), geological sciences (agriculture, environment) and civil safety (search and rescue), police operations, crowd control, and more).The report describes the analysis and assessment of the feasibility of an effective model of autonomous flying systems for monitoring agricultural and industrial sites. It demonstrates the usefulness of multirotor unmanned systems not only in popular and advertised applications of unmanned aerial vehicles in the media, but also in agriculture. The report shows an approach for automatically adapting the system to the conditions for collecting the necessary high quality data. A model has selected that uses unmanned quad-copter, hardware platform Pixhawk and ArduPilot, designed for non-commercial videos and photos of arable land.
\end{abstract}

\section{Introduction}

The development of innovation in any industry has a very positive effect on the innovation of the national economy. [1]. They are reflected, among others, in increasing expenditure on research by numerous countries' governments and offering broadly understood support for enterprises in the scope of competition, owing to the development of innovation as well as new and unique products and services [2]. The highly developed countries demonstrate the highest innovation level, as they allocate considerable funds for supporting innovative enterprises and commercialising results of research and development activities emerging based on cooperation between the science and business milieus. [3]. Therefore, we can talk about various types of innovation - technical, technological, marketing and organisational. [4]. Their operation is subject to analyses carried out by numerous scientist, such as Petrova M. and al [5], Pukala et al [6], Dyachenko et al [7], Pukala \&Adamish [8], [9-13]. They all

\footnotetext{
*Corresponding author: n.nenkov@shu.bg
} 
emphasize a unique character of such enterprises and their significant role in each country's economy.

UAVs are becoming more popular and are beginning to have actively used in various fields including agriculture. They allow for the examination in detail of agricultural land from above and has used to solve a variety of practical tasks [14]. Their implementation leads to lower costs that conventional aircraft are too large. Unlike manned aircraft, unmanned cameras are easy to use and accessible to absorb and use them. The assigned route is controlled by their computer routing and executes continuous drone commands to follow it during the flight. The effectiveness of unmanned systems increases with the number of sensors that has built into it. In the flight of the drone over objects can make images and extract useful information from them. Increasingly important for agriculture, they have functions performed by sensors that track livestock and help the shepherds control their herds and even find a lost animal. Thrones are a relatively new technology that has just entered into farming to track crop conditions, pest control and others [15].

Modern agricultural unmanned machines are fully autonomous, programmed to follow a certain trajectory. The autopilot automatically makes flying, from take-off to landing and flight with maximizing the coverage of the surveyed area and receipt of all necessary data [16]. The system automatically adapts to the conditions for collecting the necessary data quality [17]. Drone objectively offers a number of advantages over other already known methods for monitoring crops [18]:

1. Better value. For smaller fields (about 50 hectares) flight to drone out much cheaper than satellites and unmanned aircraft.

2. A high level of precision unmanned cameras captures the condition of the plants located within inches of each other due to the relatively low altitude at which monitor and provide more accurate and precise image bearing more details

3. Early detection of problems in the crops Because the drones can be used more often than other means of monitoring the farmer detects problems such as weeds, lack of water or nutrients in certain sections in time. Therefore, it may soon remove the problems and thus increase yields.

4. Full analysis capture area most commonly used method for analysing a field is a tour around the perimeter of the area to obtain information about the status of only $10-15 \%$ of the pitch with drone farmer obtains information about $100 \%$ the plot.

5.Data in 3D software allows photos be used to calculate the exact volume of hills, potholes, uneven terrain. The data has be compared with similar images of the infrared, in order to identify problems. The use of unmanned apparatus for growing crops allows farmers to receive very detailed information on the overall condition of the plantation and of individual sections, fast enough to provide for the development of crops and to react in time [19]. Therefore, in a perspective plan allows for saving resources and achieve higher yield.

When choosing a drone to monitor crops there are two possibilities:

- Multi-rotor apparatus

- Apparatus with fixed wing (airplane).

Apparatus with fixed wings fly longer and can shoot around 500 hectares, which means that they can gather more information in one flight. This in turn reduces the overall time required for data collection from a farm field. Higher efficiency leads to higher prices. Drones with fixed wings are more expensive than Multi-rotor [20]. Their prices currently vary depending on the number and type of sensors fitted. Multi-rotor devices are more suitable for small, limited areas. Because they are highly manoeuvrable, make very good 3D scanning fields and objects. Because of its manoeuvrability, however withstand twice less time in the air than those with fixed wings. They can be equipped with one to two sensors, which increases significantly the time taken to scan the entire field. The choice to 
use the technology described in a particular sector, it is important first to determine device that would suit most accurately identified needs. The application of technology drones requires investment of time and money and trained staff to work with them. Their popularity in agriculture grow, thanks to the results, which has obtained with their use. It is no secret to anyone that drones are becoming more popular. As the demand for this kind of unmanned devices increases, logically grow and demand.

\section{Results and Discussion}

Nowadays the development of unmanned machines is not so difficult. The market for electronics has a wide selection of hardware components and modules suitable for the production of an unmanned system. From a software perspective, the possibility of establishing such a system, designed to perform specific functions are extremely limited. Most manufacturers use software developed and implemented only with two types of apparatus (plane or multirotor), with the support of most sensors is limited, which gives a great advantage of the apparatus working with open source as ArduPilot example.

Table 1. Hardware features of both devices

\begin{tabular}{|c|c|c|}
\hline Characteristic & Pixhawk/Ardu-Copter & DJI Phantom 3 \\
\hline Weight & 2600 grams & 1280 grams \\
\hline GNSS & GPS/GLONASS & GPS/GLONASS \\
\hline Max speed & $22 \mathrm{~m} / \mathrm{c}$ & $16 \mathrm{~m} / \mathrm{c}$ \\
\hline Capacity of battery & $25.20 \mathrm{v} / 12000 \mathrm{mAh}$ & $15.2 \mathrm{~V} / 4480 \mathrm{mAh}$ \\
\hline Time on flight & $30 \mathrm{~min}$ & $20 \mathrm{~min}$ \\
\hline Camera & Xiaomi Yi/ $16 \mathrm{pix}$ & $\mathrm{DJ} / 12.76 \mathrm{pix}$ \\
\hline Captured area & $350 \mathrm{ha}$ & $200 \mathrm{ha}$ \\
\hline Range of radio tech & $3000 \mathrm{~m}$ & $1500 \mathrm{~m}$ \\
\hline
\end{tabular}

The advantages of using the ArduPilot open source system are:

- Price - Varies with hardware configuration. Management software, configuration, planning- free.

- Modularity- support of a number of sensors and modules.

- Multifunctionality- use project in three separate types of apparatus applicable in agriculture (scout drone-aircraft drone scout multi-copter, drone sprayer). One of the biggest advantages of the system is that all sensors used in three types of apparatus are interchangeable.

- Ability to unlimited range of connection between devices and ground station (including video signal) via TCP / IP, UDP, 3G, 4G with MAVLink communication protocol. Management sets of radio equipment or the ground station via a joystick.

- Support project software tracker antennas is an invaluable tool in performing tasks related to flight over a large territory

- Software batter missions with extraordinary opportunities and working on all mobile devices.

- Use of different types of batteries.

Quad-copter developing an unmanned system hardware platform Pixhawk and ArduPilot intended (see Table 1) for non-commercial video and photo shooting can easily compare it with a similar one, ready to use DJI Phantom 3, both sets will be used to capture cultivated areas. It is important to note that both have drone it captured only in RGB spectrum for the purpose of study photographs should be treated with VARI (Visible Atmospherically Resistant Index) algorithm [21].

Prominent characteristics of both sets, modelling machine has better parameters for carrying out their tasks. The fact is that the modelling is well-appreciated ratio between 
power and payload [17]. Estimated is the need to add additional load, additional sensors for further research, peripheral modules and a more powerful battery for long flight (see fig 1).

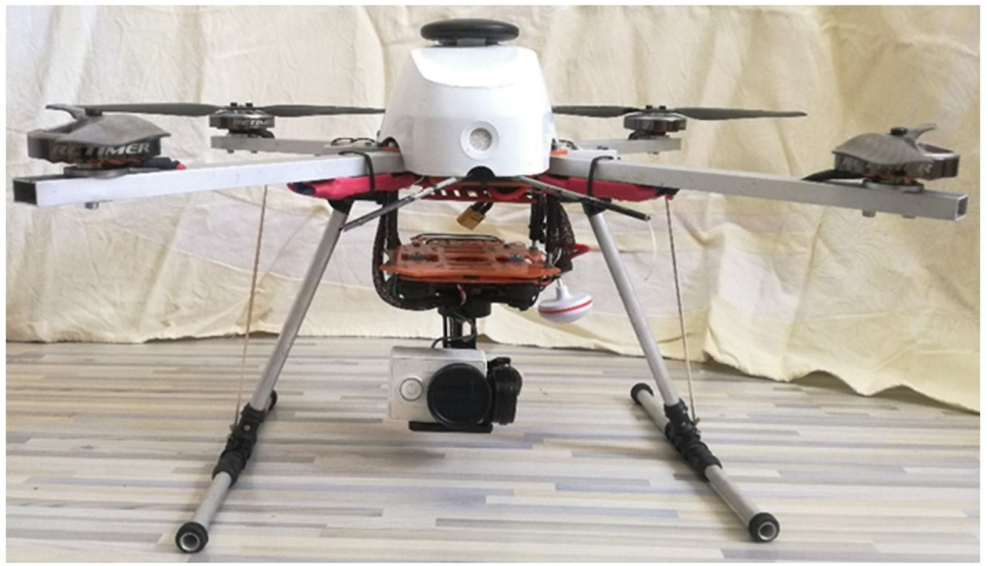

Fig. 1. Quadcopter system hardware platform Pixhawk and ArduPilot.

Both camera shoot cultures in the RGB spectrum of light, but to have maximum correctness photos filming should be done with special cameras and filters with a relatively affordable price. Seen from another perspective possible modification of the cameras of both the drone by replacing factory modified their lenses, which is also an affordable price. After the tests carried, out with the modified chambers of both drones has achieved similar and satisfactory results (see fig 2).

The only advantage of DJI Phantom 3 in a hardware aspect is the automatic addition of geolocation data (location and height) in the pictures. The camera used with the model camera does not have this feature, which complicates post-data processing with additional software processing.

One of the reasons "agro drone" are so expensive are invested in them sensors and cameras that are $2 / 3$ the price of the device. Another reason these expensive systems is software for mission planning and software for data processing. In most cases, purchasing machinery or specialized camera monitoring crop, the manufacturer provides you with software for processing the collected information, which forces you to invest further in computers with better performance. When working with modified cameras is the right option to use, the services of online-based platforms such as drone deploy, where information has treated with the necessary study filter algorithms [21-22].

It is not necessary to purchase a license for a whole year because, actually monitoring lasts about six months. The platform offers a number of tools for pro-cessing information collected in the form of applications that compile detailed reports on the processed data (number of crops, presence of birds, report damage from disasters, etc.). One of the main things that are different the two systems is software for device management and planning missions. 


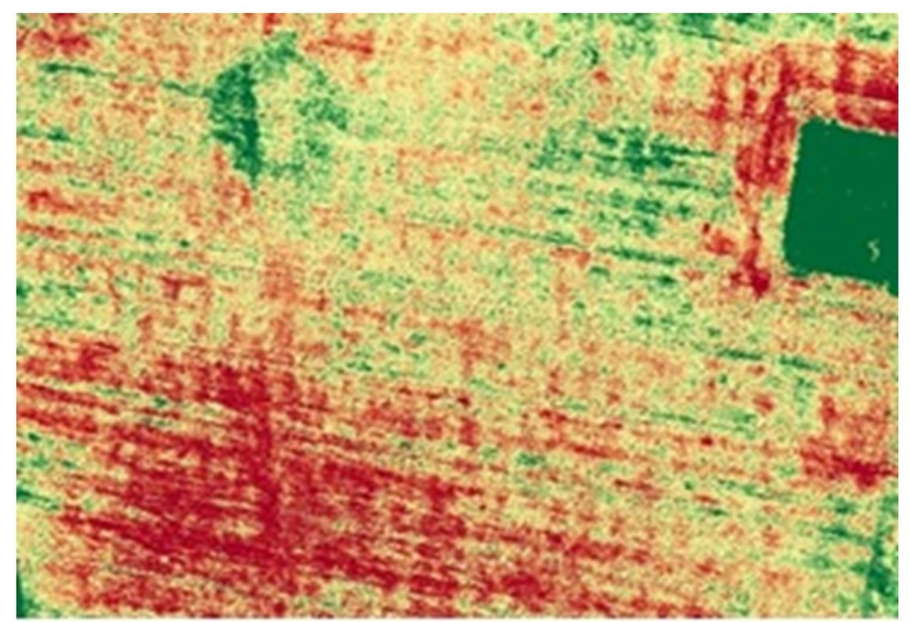

Fig. 2. NDVI picture of the test field.

The modelled device works with mobile and stationary devices, applications planned missions are open source and completely free. Route planning has enhanced with a multitude of tools that automatically create appropriate for the purposes specified plan, consistent with the characteristics of the sensors monitoring. It is necessary to mention that code ArduPilot is multifunctional and has be used in other robotic devices, which are in support of agriculture (drone sprayer aircraft). Developing three types of unmanned systems in an economy based on ArduPilot project, we are full advantage of the opportunities smart agriculture: airplane for use in large areas, quad-copter small and drone sprayer accurate machining after problems found [23-24].

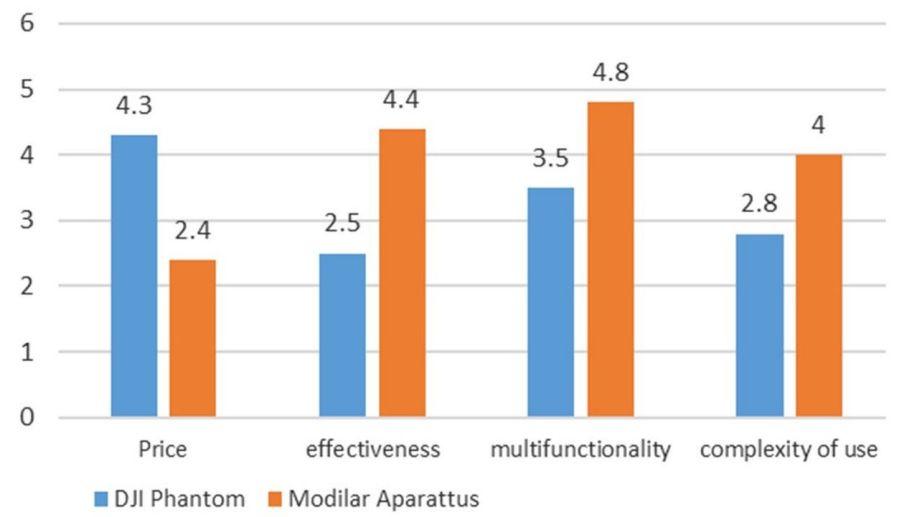

Fig. 3. Comparison between modelled and apparatus DJI Phantom3 used to agro monitoring.

All devices has be controlled from a ground station. When using drone DJI Phantom series has forced to use only mobile devices that use paid software for route planning and have extremely limited opportunities. Company policy to limit the height to $30 \mathrm{~m}$. In addition, length up to $50 \mathrm{~m}$. In flight without connected mobile device further restricts the use of these devices for these purposes.

The outcome of this development has created "budget" unmanned aircraft to autonomously on missions ArduPilot. The main objective of the drone stage capture farmland and production of "health card" of crops as shown in Figure 1. A comparison of 
four indicator as shown in Fig. 3 shows the advantage of ArduPilot front of a commercial drone DJI Phantom.

\section{Conclusion}

The article demonstrates the usefulness of multi-rotor unmanned systems, not only in popular and advertised applications of drones in the media, but also in agriculture. Demonstrate the possibilities to create a relatively inexpensive system that is reliable, hightech and very useful device equipped with GPS navigation, sensors and cameras for aerial photography in high resolution. ArduPilot allows organizing precision farming based on much greater accuracy of satellite data and much less costly than for manned surveillance used in the past. In modeling, they solved many techno-logical tasks such as the choice of platform and code management, apparatus design, component selection, assembly and configuration of the system. Platform is multifunctional and provides choices for adding and managing the various modules. Eventually created a system that can carry different types of cameras and sensors, as well as to deliver consignments sprayed crops and other useful activities for agricultural purposes. After the test the system, it is found that can easily be deployed in agriculture in preference to use expensive methods to capture of satellites and planes. The cards collected by unmanned cameras then turn into a "recipe for treatment" for the area. They in turn can get on a tractor or other machine with GPSnavigation that ensure delivery of the required amount of fertilizers, pesticides or other resources.

\section{References}

1. R. Pukala, Economics and Management, 8:3, 43 (2016)

2. N. Kurmanov, B. Tolysbayev, D. Aibossynova, \& N. Parmanov, Economic Annals-XXI, 158:3, (2016)

3. N. Kurmanov, D. Turekulova, G. Doskeyeva, \& G. Alina, International Journal of Economics and Financial Issues, 6:3, 907 (2016)

4. T. Uteubayev, International Journal of Economics and Financial Issues, 6:4, 1419 (2016)

5. M. Petrova, , O. Sushchenko, , I. Trunina, N. Dekhtyar, IEEE First International Conference on System Analysis \& Intelligent Computing (SAIC-2018) (2018)

6. R. Pukała, P. Adamisin, R. Vavrek, 15-th International Multidisciplinary Scientific Geo conference SGEM 2015, 18 (2015)

7. Y. Dyachenko, N. Nenkov, M. Petrova, I. Skarga-Bandurova, O. Soloviov, Biologically Inspired Cognitive Architectures, 26, 130 (2018)

8. R. Pukala, S. Kaidarova, S. Makysh, K. Zhumanov, 31st International-Business-InformationManagement-Association Conference (2018)

9. M. Petrova, M. Tepavicharova, L. Boykova, E3S Web of Conferences, 41, 04017 (2018)

10. M. Petrova, N. Dekhtyar, O. Klok, O. Loseva, Problems and Perspectives in Management, 16:4, $259(2018)$

11. V. Koval, G. Duginets, O. Plekhanova, A. Antonov, M. Petrova Entrepreneurship and Sustainability Issues, 6:4, (2019)

12. T. Odinokova, M. Bozhinova, M. Petrova, E3S Web Conferences, 41, 04015 (2018)

13. M. Petrova, , O. Sushchenko, , I. Trunina, N. Dekhtyar, IEEE First International Conference on System Analysis \& Intelligent Computing (SAIC-2018)) 256 (2018)

14. Agro Technology - plant growing, livestock, bioenergya (2016)

15. Agriculture Drone Business Takes Off: (2017)

16. Agriculture Drone Business Takes Off (2017)

17. Advanced Multi-Copter (2017)

18. Drone life, 5 Tips for Evaluating Online Drone Data Services: (2016) 
19. Q. Quan, Introduction to Multicopter Design and Control (2018)

20. T. Kilby, B . Kilby, Drony with Zero, (BHV-Petersburg, Petersburg, 2016)

21. K. Nonami, F. Kendoul, S. Suzuki, W. Wang, D. Nakazawa, Autonomous Flying Ro-bots Unmanned Aerial Vehicles and Micro Aerial Vehicles (2018)

22. N.V. Nenkov, Computer Modelling \& New Technologies (Riga, Latvia, 2015)

23. Work Smarter with Drone (2018)

24. J. Pleban, R. Band, R. Creutzburg, IS\&T/SPIE Electronic Imaging. In-ternational Society for Optics and Photonics (2014) 